OPEN ACCESS

Edited by:

Jordi Pérez-Tur

Consejo Superior de Investigaciones

Científicas (CSIC), Spain

Reviewed by:

Yuan Gao,

Shandong University, China

Miaojin Zhou,

Central South University, China

*Correspondence:

Yuedong Wang

2112014@zju.edu.cn

Specialty section:

This article was submitted to Genetics of Common and Rare

Diseases,

a section of the journal

Frontiers in Genetics

Received: 05 March 2021 Accepted: 15 June 2021

Published: 09 July 2021

Citation:

Zhan X, He L, Song K, Cao S, Meng E and Wang Y (2021) Case Report:

Triple Primary Malignant Tumors of the Esophagus, Stomach, and Colon in a Patient With Genetic Analysis.

Front. Genet. 12:676497.

doi: 10.3389/fgene.2021.676497

\section{Case Report: Triple Primary Malignant Tumors of the Esophagus, Stomach, and Colon in a Patient With Genetic Analysis}

\author{
Xiaoli Zhan ${ }^{1}$, Lingzhe $\mathrm{He}^{1}$, Kai Song ${ }^{2}$, Shuliang $\mathrm{Cao}^{3}$, Erhong Meng ${ }^{3}$ and Yuedong Wang ${ }^{1 \star}$ \\ ${ }^{1}$ Department of General Surgery, The Second Affiliated Hospital Zhejiang University School of Medicine, Hangzhou, China, \\ ${ }^{2}$ Department of Pathology, The Second Affiliated Hospital Zhejiang University School of Medicine, Hangzhou, China, \\ ${ }^{3}$ ChosenMed Technology (Beijing) Co. Ltd., Beijing, China
}

The incidence of multiple primary malignant tumors (MPMTs) has increased greatly with the progress of tumor diagnosis and therapy technology. However, triple primary cancer is still very rare, and its genetic change is not clear yet. This case report described a 70-year-old Chinese male patient with triple primary cancers of the esophagus, stomach and right-sided colon. Pathological examination confirmed that each malignant tumor developed independently. Next-generation sequencing (NGS) using a 599-gene panel revealed five TP53 mutations in three tumor tissues. These variations might contribute to development of the triple primary malignant tumors in the patient. The patient underwent laparoscopic feeding jejunostomy and postoperative radiotherapy for synchronous esophageal and gastric carcinomas. Then, he underwent laparoscopic-assisted resection of right-sided colonic cancer and lysis of abdominal adhesions. By the time of submitting this manuscript, the patient had been well and no sign of recurrence or metastasis had been observed. To the best of our knowledge, this case is the first one to clarify the genetic abnormalities of triple primary cancers of esophagus, stomach and colon in a Chinese patient. It may contribute to understanding the molecular pathogenesis of multiple primary digestive malignancies and providing valuable treatment strategies for the similar patients in the future.

Keywords: multiple primary malignant tumors, esophagus cancer, gastric cancer, colon cancer, genetics

\section{BACKGROUND}

With the development of tumor diagnosis and treatment technology, the incidence of multiple primary malignant tumors (MPMTs) has increased greatly (Kumagai et al., 2001). However, triple primary cancer is still a very rare finding, and its genetic change is not clear yet. In this study, we reported the case of a 70-year-old Chinese man with triple primary cancers of esophagus, stomach and right-sided colon and clarified the genetic abnormalities underlying them.

\section{CASE PRESENTATION}

A 70-year-old male patient was admitted to our hospital complaining of choking while eating for more than 1 month on March 20, 2019. CT showed tumor signs in the middle of esophagus 
A

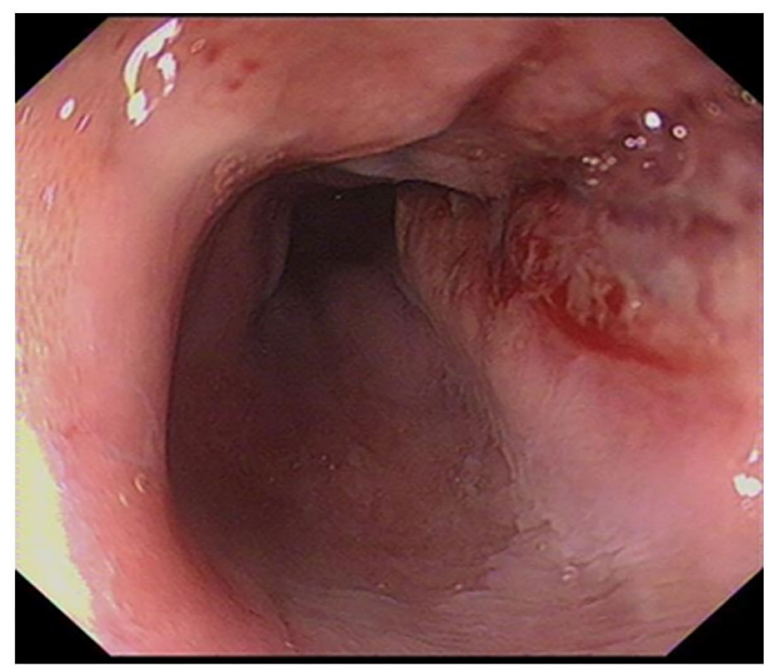

c

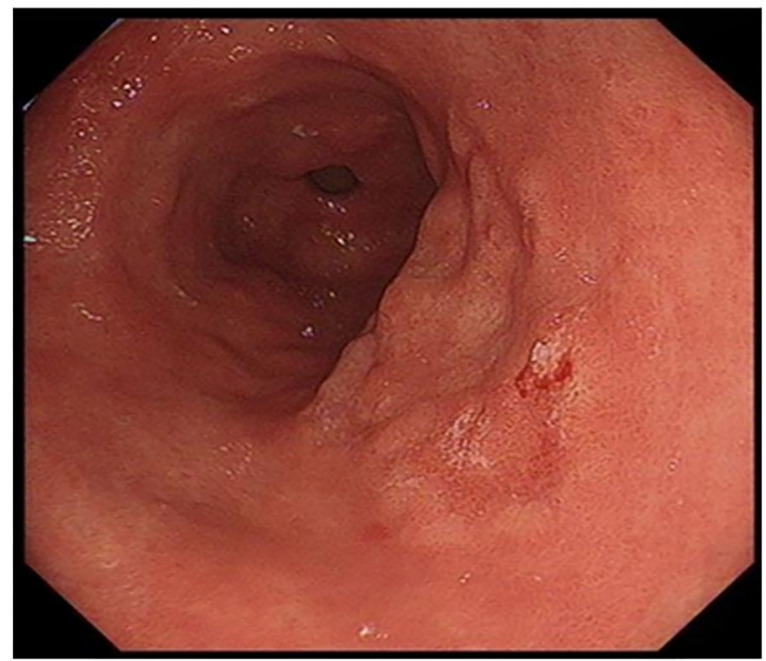

E

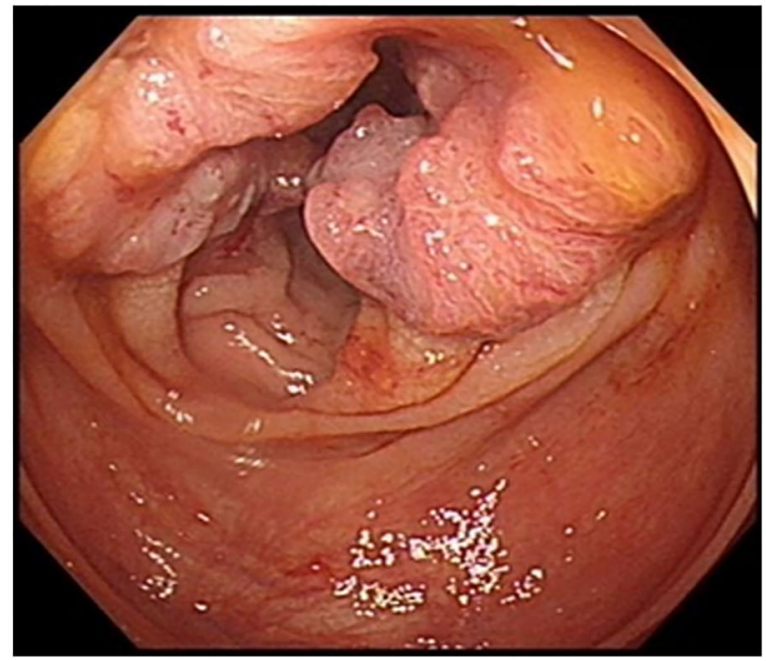

B

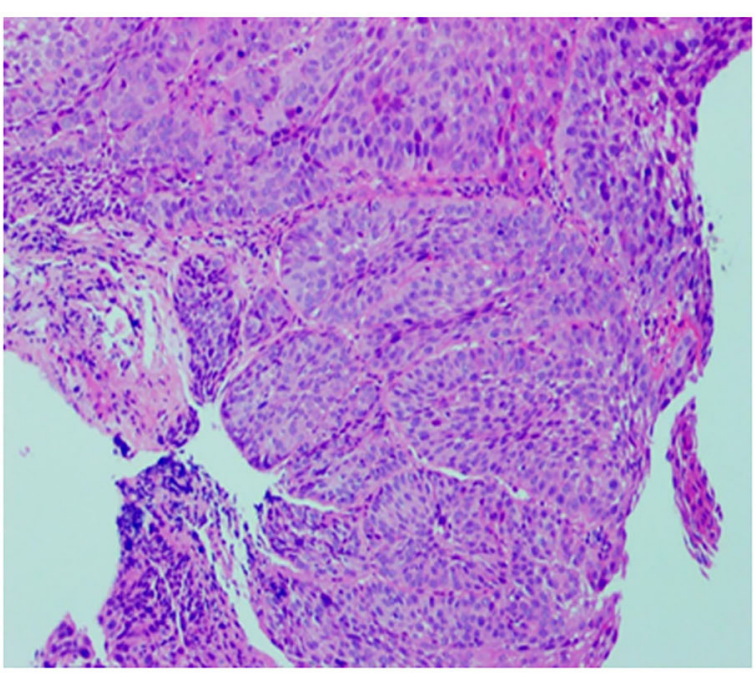

D

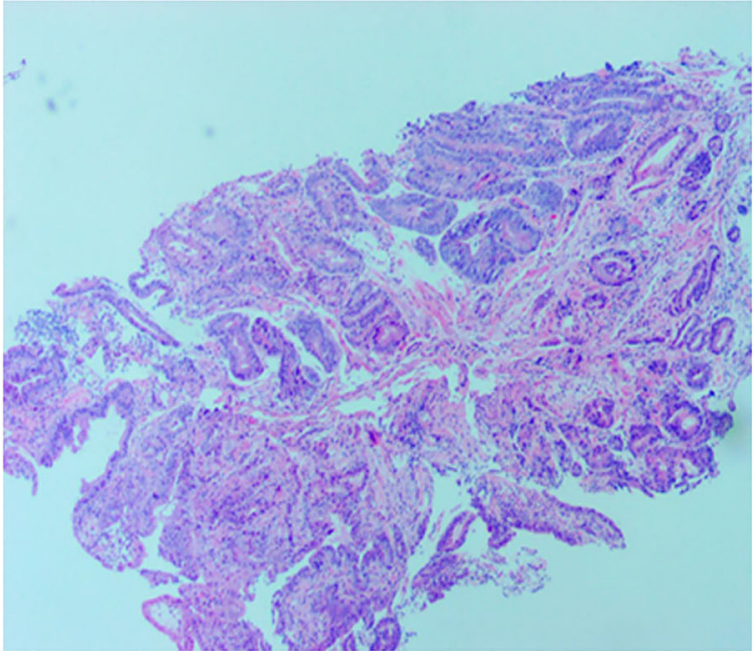

$\mathbf{F}$

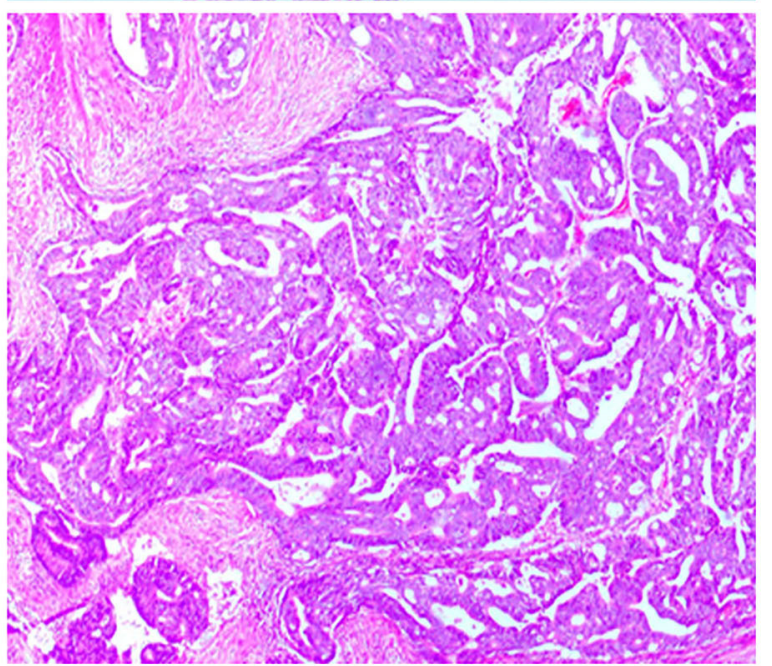

FIGURE 1 | Esophagogastroduodenoscopic, Colonoscopic, and microscopic morphology of esophageal carcinoma, gastric carcinoma as well as colon cancer. (A) Esophagogastroduodenoscopic morphology of esophageal carcinoma. (B) Pathological examination showed esophageal squamous cell carcinoma (H.E. $\times$ 100). (C) Esophagogastroduodenoscopic morphology of gastric carcinoma. (D) Pathological examination revealed well-differentiated gastric adenocarcinoma (H.E. $\times$ 100). (E) Colonoscopic morphology of right-sided colon. (F) Pathological examination showed moderately differentiated adenocarcinoma of colon (H.E. $\times 100)$. 


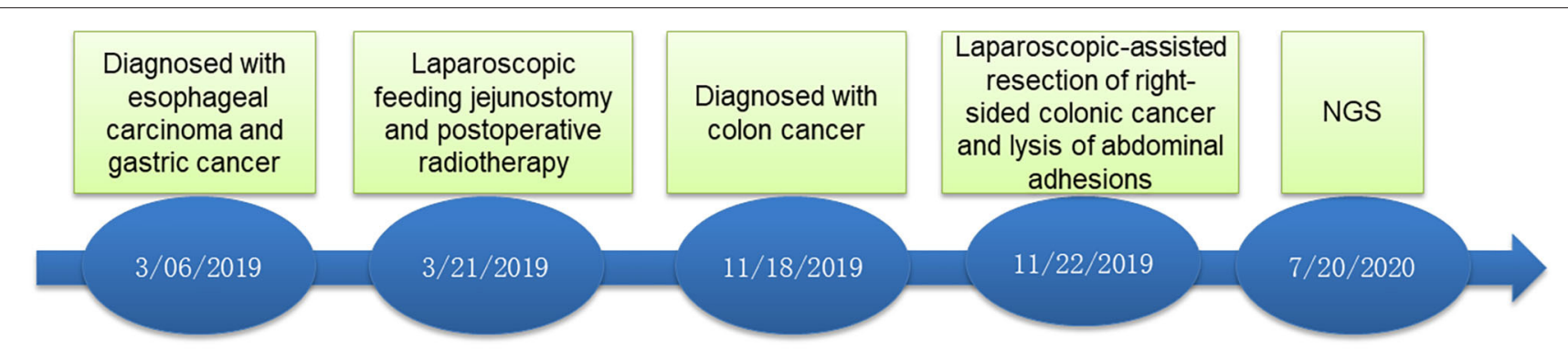

FIGURE 2 | Timeline of the patient.

on March 6, 2019. Esophagogastroduodenoscopy demonstrated the patient was suffering from esophageal carcinoma and gastric cancer (Figures 1A,C). He had no family history of malignancy. Pathology examination confirmed they were locally advanced esophageal squamous cell carcinoma and adenocarcinoma of gastric horn (Figures 1B,D). On March 21, 2019, the patient underwent laparoscopic feeding jejunostomy and postoperative radiotherapy.

On November 15 and 18, 2019, esophagogastroduodenoscopy and abdominal CT showed that esophageal and gastric cancers were under control. However, CT indicated a tumor in the right-sided colon. On November 19, 2019, colonoscopy and pathological findings confirmed it was locally advanced moderately differentiated adenocarcinoma of colon (Figures 1E,F). Immunohistochemical stains revealed proficient mismatch repair proteins and positive expression of S-100, but negative expression of D2-40 or CD34. The patients underwent laparoscopic-assisted resection of rightsided colonic cancer and lysis of abdominal adhesions on November 22, 2019. By the time of submission of this manuscript, the patient had been well and no sign of recurrence or metastasis had been detected. The timeline was shown as Figure 2.

In order to seek possible personalized therapy strategies, the paraffin-embedded tissue blocks from malignant esophagus, stomach and right-sided colon, respectively, were subjected to next-generation sequencing (NGS) using a pan-cancer 599gene panel [ChosenOne599, ChosenMed Technology (Beijing) Co. Ltd, Beijing, China] on July 20, 2020. NGS demonstrated the TMB value was 10.11 Muts/Mb, 11.37 Muts/Mb, 6.32 Muts/Mb in the tumor tissues of esophagus, stomach and colon, respectively, which ranked $29.58 \%$ (moderate), $6.45 \%$ (high) and $62.73 \%$ (low) in patients with corresponding malignancy. TP53 mutation was identified in all three tumor tissues. However, the mutation location was different from each other. In the esophagus cancer tissues, two TP53 variations were identified (NM_000546: exon6: c.580C>T: p.L194F and NM_000546: exon8: c.814G $>$ A: p.V272M). In the gastric carcinoma tissues, there were also two TP53 alterations (NM_000546: exon4: c.375G >A: p.T125T and NM_000546: exon7: c.715A $>$ G: p.N239D). In the right-sided colon carcinoma tissues, there was one TP53 mutation (NM_000546: exon8: c.821T>G: p.V274G) (Table 1).
At the same time, other important variations were also identified in the esophagus carcinoma tissues, including ATRX (NM_000489: exon21: c.5424T>G: p.Y1808*), CHEK1 (NM_001114121: exon7: c.676dup: p.T226fs), copy number variations (CNVs) of CCND1 and FGF3/4/19. In the gastric carcinoma tissues, the same CHEK1 variation was also detected. In the colon carcinoma tissues, there were two $A P C$ variations (NM_000038: exon16: c.4468del: p.H1490fs and NM_000038: exon16: c.2621C>G: p.S874*, Table 1).

\section{DISCUSSION}

MPMTs are referred to the presence at least two histologically distinct malignancies that are not caused by recurrence or metastasis in the same patient. For the diagnosis of two primary malignant tumors, the following conditions must be met: (Dranka-Bojarowska and Lewinski, 2019) (a) Pathology confirms that both are malignancies; (b) Malignancies must locate separately. If they are close, they must be separated by a healthy mucosa with an area of at least $2 \mathrm{~cm}$. If they are formed in the same organ, the period of more than 5 years after diagnosis must pass; (c) The possibility that the second malignancy is caused by metastasis from the primary site is excluded.

There are two kinds of MPMTs: synchronous and metachronous MPMTs. The former is defined as the diagnosis of a second primary malignancy within 6 months of the first primary malignancy, while the latter refers to the second primary malignancy diagnosed over 6 months after the first primary one (Zhai et al., 2018). Studies revealed that MPMTs often occur in the digestive system, followed by digestive-respiratory system in China (Zhai et al., 2018). The patient in our study was firstly diagnosed as esophagus carcinoma, then as gastric adenocarcinoma within one month (synchronously), finally as colon cancer 8 months later (metachronously).

To date, the molecular pathogenesis of MPMTs remains elusive. Some studies speculated that the development of MPMTs was associated with unhealthy lifestyle, genetic susceptibility, side effects of chemotherapy and radiotherapy, weak immunity, etc. The patient had a long history of smoking and drinking, and it might be one of predisposing factors of his MPMTs.

Studies demonstrated cancer develops from the accumulation of mutations in oncogenes and tumor suppressor genes (Califano et al., 1996). It is in line with this study, as the NGS data demonstrated the TMB value was middle to high, i.e., 10.11 
TABLE 1 | Genetic alterations detected in the patient's tumor tissues.

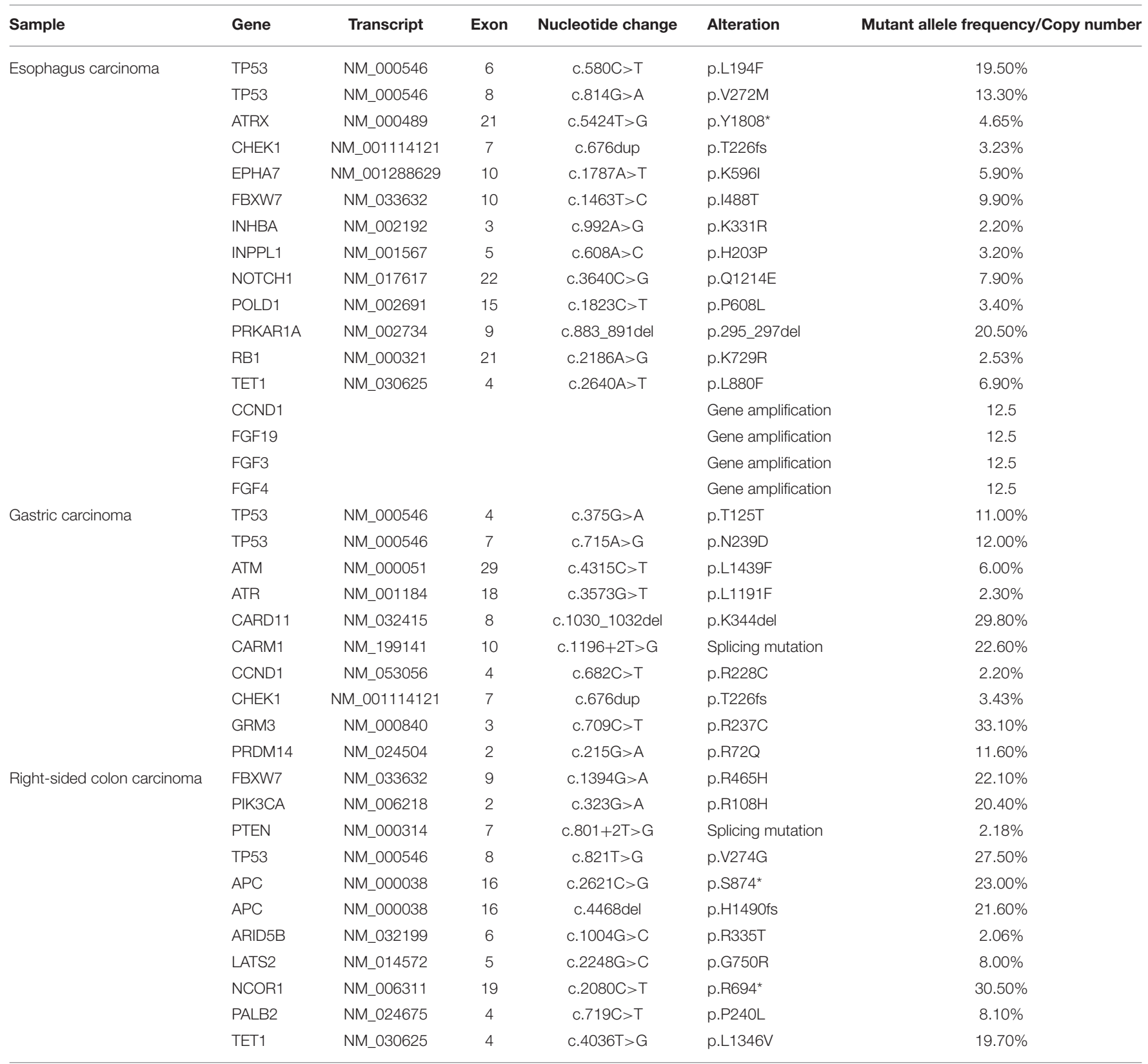

Muts/Mb, 11.37 Muts/Mb, 6.32 Muts/Mb in the tumor sample of esophagus, stomach and colon, respectively, which ranked 29.58, 6.45 , and $62.73 \%$ in the patients with corresponding malignancy.

Study of Dranka-Bojarowska and Lewinski (2019) revealed that genetic background, especially mutation of tumor suppressor gene TP53, BRCA1-associated protein 1 (BAP1) (Cheung et al., 2015) or partner and localizer of BRCA2 (PALB2) (Schrader et al., 2016) played a crucial role in the development of MPMTs. Germline TP53 mutation is closely associated with Li-Fraumeni Syndrome, characterized by a high frequency of various malignancies (Consul et al., 2020). In the patient, five different TP53 mutations were detected in the patient's three tumors samples. Currently, clinical trial on Adavosertib (AZD1775, NCT02448329) targeted against TP53 variations is ongoing.

In addition, ATRX variation (NM_000489: exon21: c. $5424 \mathrm{~T}>\mathrm{G}$ : p.Y1808*) was also detected in the esophagus carcinoma tissues. According to the annotations of OncoKB, the ATRX mutation may be pathogenic. Previous studies have shown that loss of ATRX is associated with a variety of tumors such as sarcomas, hepatic angiosarcomas, gliomas and so on (de Wilde et al., 2012; Marinoni et al., 2014; Qadeer et al., 2014; Leeper et al., 2015; Liau et al., 2015a,b,c; Rodriguez et al., 2016; Singhi 
et al., 2017). There is currently no targeted therapy against the ATRX mutation.

The patient harbored CHEK1 (NM_001114121: exon7: c.676dup:p.T226fs) in the tissues of esophagus carcinoma and gastric carcinoma (Table 1), Previous studies have shown that CHEK1 mutation was associated with multiple malignancies, including lung cancer, prostate cancer, cervical cancer, and colorectal cancer (Gali-Muhtasib et al., 2008; Mazumder Indra et al., 2011; Al Nakouzi et al., 2014; Liu et al., 2015). According to the annotations of OncoKB, the CHEK1 mutation may lead to loss of protein function, which is possibly pathogenic. However, no targeted therapy against the CHEK1 mutation has been approved.

Besides, the patient also harbored CNVs of CCND1 and FGF3/4/19 (Table 1). CCND1 often co-amplified with FGF3/4/19. A study reported that $86.7 \%(26 / 30)$ of the patients with esophageal carcinoma showed positive expression of CCND1 through immunohistochemistry (Hu et al., 2016). Clinical trials on Palbociclib (NCT04439201) and Abemaciclib (NCT03356223) targeted against CCND1 amplification are ongoing.

In the colon carcinoma tissues, two $A P C$ alterations were detected (Table 1). According to the annotations of OncoKB, both APC mutations could cause protein loss of function, abnormal $\mathrm{Wnt} / \beta$-catenin signaling pathway and promote the development of colon cancer. To date, No targeted drug against these two APC mutations have been approved.

Germline mutations were not mentioned in this study because none of the patient's family members had malignant tumors.

The treatment strategies for MPMTs depend on some factors, such as the tumor stage, patient's age and comorbidities. Furthermore, a multidisciplinary team management approach is necessary for personalized therapy. In metachronous MPMTs, the treatment always involves sequential therapy for each tumor, whereas in synchronous MPMTs, individualized and single treatment is decided upon after appropriate evaluation and consensus is reached by multidisciplinary team (Vogt et al., 2017). The patient underwent surgery and postoperative radiotherapy for synchronous esophageal and gastric carcinomas. Then, he underwent laparoscopic-assisted resection of rightsided colonic cancer and lysis of abdominal adhesions. By the time of submitting this manuscript, the patient had

\section{REFERENCES}

Al Nakouzi, N., Cotteret, S., Commo, F., Gaudin, C., Rajpar, S., Dessen, P., et al. (2014). Targeting CDC25C, PLK1 and CHEK1 to overcome Docetaxel resistance induced by loss of LZTS1 in prostate cancer. Oncotarget 5, 667-678. doi: 10.18632/oncotarget.1574

Califano, J., van der Riet, P., Westra, W., Nawroz, H., Clayman, G., Piantadosi, S., et al. (1996). Genetic progression model for head and neck cancer: implications for field cancerization. Cancer Res. 56, 2488-2492.

Cheung, M., Kadariya, Y., Talarchek, J., Pei, J., Ohar, J. A., Kayaleh, O. R., et al. (2015). Germline BAP1 mutation in a family with high incidence of multiple primary cancers and a potential gene-environment interaction. Cancer Lett. 369, 261-265. doi: 10.1016/j.canlet.2015.09.011 been well and no sign of recurrence or metastasis had been detected.

To the best of our knowledge, this case is the first one to clarify the genetic abnormalities of triple primary cancers of esophagus, stomach and colon in a Chinese patient. It may contribute to understanding the molecular pathogenesis of multiple primary digestive malignancies and providing valuable treatment strategies for the similar patients in the future. The limitation of this study is that NGS-based multi-gene panel couldn't identify all genetic predispositions like epigenetic modifications and other CNVs.

\section{DATA AVAILABILITY STATEMENT}

All of the data supporting the findings in this study are available upon reasonable request from the corresponding author (Yuedong Wang).

\section{ETHICS STATEMENT}

The studies involving human participants were reviewed and approved by the Ethics Committee of the Second Affiliated Hospital Zhejiang University School of Medicine. Written informed consent for participation in the study, publication of clinical details and images was obtained from the patient.

\section{AUTHOR CONTRIBUTIONS}

YW: conceptualization. XZ: writing-original draft. SC and EM: conducting bioinformatics analysis. LH and KS: writing-review and editing. All authors contributed to the article and approved the submitted version.

\section{ACKNOWLEDGMENTS}

The authors thank the patient and all of personnel who participated in this study.

\section{SUPPLEMENTARY MATERIAL}

The Supplementary Material for this article can be found online at: https://www.frontiersin.org/articles/10.3389/fgene. 2021.676497/full\#supplementary-material 
Gali-Muhtasib, H., Kuester, D., Mawrin, C., Bajbouj, K., Diestel, A., Ocker, M., et al. (2008). Thymoquinone triggers inactivation of the stress response pathway sensor CHEK1 and contributes to apoptosis in colorectal cancer cells. Cancer Res. 68, 5609-5618. doi: 10.1158/0008-5472.CAN-08-0884

Hu, X., Moon, J. W., Li, S., Xu, W., Wang, X., Liu, Y., et al. (2016). Amplification and overexpression of CTTN and CCND1 at chromosome 11q13 in Esophagus squamous cell carcinoma (ESCC) of North Eastern Chinese Population. Int. J. Med. Sci. 13, 868-874. doi: 10.7150/ijms.16845

Kumagai, Y., Kawano, T., Nakajima, Y., Nagai, K., Inoue, H., Nara, S., et al. (2001). Multiple primary cancers associated with esophageal carcinoma. Surg. Today 31, 872-876. doi: 10.1007/s005950170025

Leeper, H. E., Caron, A. A., Decker, P. A., Jenkins, R. B., Lachance, D. H., and Giannini, C. (2015). IDH mutation, 1p19q codeletion and ATRX loss in WHO grade II gliomas. Oncotarget 6, 30295-30305. doi: 10.18632/oncotarget.4497

Liau, J. Y., Lee, J. C., Tsai, J. H., Yang, C. Y., Liu, T. L., Ke, Z. L., et al. (2015a). Comprehensive screening of alternative lengthening of telomeres phenotype and loss of ATRX expression in sarcomas. Mod. Pathol. 28, 1545-1554. doi: 10.1038/modpathol.2015.114

Liau, J. Y., Tsai, J. H., Jeng, Y. M., Lee, J. C., Hsu, H. H., and Yang, C. Y. (2015b). Leiomyosarcoma with alternative lengthening of telomeres is associated with aggressive histologic features, loss of ATRX expression, and poor clinical outcome. Am. J. Surg. Pathol. 39, 236-244. doi: 10.1097/PAS.0000000000000324

Liau, J. Y., Tsai, J. H., Yang, C. Y., Lee, J. C., Liang, C. W., Hsu, H. H., et al. (2015c). Alternative lengthening of telomeres phenotype in malignant vascular tumors is highly associated with loss of ATRX expression and is frequently observed in hepatic angiosarcomas. Hum. Pathol. 46, 1360-1366. doi: 10.1016/j.humpath.2015.05.019

Liu, B., Qu, J., Xu, F., Guo, Y., Wang, Y., Yu, H., et al. (2015). MiR-195 suppresses non-small cell lung cancer by targeting CHEK1. Oncotarget 6, 9445-9456. doi: 10.18632/oncotarget.3255

Marinoni, I., Kurrer, A. S., Vassella, E., Dettmer, M., Rudolph, T., Banz, V., et al. (2014). Loss of DAXX and ATRX are associated with chromosome instability and reduced survival of patients with pancreatic neuroendocrine tumors. Gastroenterology 146, 453 e5-460 e5. doi: 10.1053/j.gastro.2013.10.020

Mazumder Indra, D., Mitra, S., Singh, R. K., Dutta, S., Roy, A., Mondal, R. K., et al. (2011). Inactivation of CHEK1 and EI24 is associated with the development of invasive cervical carcinoma: clinical and prognostic implications. Int. J. Cancer 129, 1859-1871. doi: 10.1002/ijc.25849
Qadeer, Z. A., Harcharik, S., Valle-Garcia, D., Chen, C., Birge, M. B., Vardabasso, C., et al. (2014). Decreased expression of the chromatin remodeler ATRX associates with melanoma progression. J. Invest. Dermatol. 134, 1768-1772. doi: 10.1038/jid.20 14.45

Rodriguez, F. J., Vizcaino, M. A., Blakeley, J., and Heaphy, C. M. (2016). Frequent alternative lengthening of telomeres and ATRX loss in adult NF1-associated diffuse and high-grade astrocytomas. Acta Neuropathol. 132, 761-763. doi: 10.1007/s00401-016-1 619-0

Schrader, K. A., Stratton, K. L., Murali, R., Laitman, Y., Cavallone, L., Offit, L., et al (2016). Genome sequencing of multiple primary tumors reveals a novel PALB2 variant. J. Clin. Oncol. 34, e61-e67. doi: 10.1200/JCO.2013.50.0272

Singhi, A. D., Liu, T. C., Roncaioli, J. L., Cao, D., Zeh, H. J., Zureikat, A. H., et al. (2017). Alternative lengthening of telomeres and loss of DAXX/ATRX expression predicts metastatic disease and poor survival in patients with pancreatic neuroendocrine tumors. Clin. Cancer Res. 23, 600-609. doi: 10.1158/1078-0432.CCR-16-1113

Vogt, A., Schmid, S., Heinimann, K., Frick, H., Herrmann, C., Cerny, T., et al. (2017). Multiple primary tumours: challenges and approaches, a review. ESMO Open 2:e000172. doi: 10.1136/esmoopen-2017-000172

Zhai, C., Cai, Y., Lou, F., Liu, Z., Xie, J., Zhou, X., et al. (2018). Multiple primary malignant tumors-a clinical analysis of 15,321 patients with malignancies at a single center in China. J. Cancer 9, 2795-2801. doi: 10.7150/jca.25482

Conflict of Interest: SC and EM are employees at ChosenMed Technology.

The remaining authors declare that the research was conducted in the absence of any commercial or financial relationships that could be construed as a potential conflict of interest.

Copyright (C) 2021 Zhan, He, Song, Cao, Meng and Wang. This is an open-access article distributed under the terms of the Creative Commons Attribution License (CC $B Y)$. The use, distribution or reproduction in other forums is permitted, provided the original author(s) and the copyright owner(s) are credited and that the original publication in this journal is cited, in accordance with accepted academic practice. No use, distribution or reproduction is permitted which does not comply with these terms. 